\title{
DNA molecules and configurations in a solid-state nanopore microscope
}

\section{Citation}

Li, Jiali, Marc Gershow, Derek Stein, Eric Brandin, and J. A. Golovchenko. 2003. “DNA Molecules and Configurations in a Solid-State Nanopore Microscope." Nat Mater 2 (9) (August 24): 611-615. doi: $10.1038 /$ nmat 965 .

\section{Published Version}

doi:10.1038/nmat965

\section{Permanent link}

http://nrs.harvard.edu/urn-3:HUL.InstRepos:27877632

\section{Terms of Use}

This article was downloaded from Harvard University's DASH repository, and is made available under the terms and conditions applicable to Other Posted Material, as set forth at http:// nrs.harvard.edu/urn-3:HUL.InstRepos:dash.current.terms-of-use\#LAA

\section{Share Your Story}

The Harvard community has made this article openly available.

Please share how this access benefits you. Submit a story.

Accessibility 


\section{DNA Molecules and Configurations in a}

\section{Solid-State Nanopore Microscope}

Jiali Li*§, Marc Gershow*, Derek Stein†\#, Eric Brandin†, and J. A. Golovchenko*†

*Department of Physics, †Division of Engineering and Applied Sciences, and $\ddagger$ Department of Molecular and Cellular Biology, Harvard University, Cambridge, Massachusetts 02138, USA

§Present address: Department of Physics, University of Arkansas, Fayetteville, Arkansas 72701, USA; \#Present address: Department of NanoScience, Delft University of Technology, Lorentzweg 1, 2628 CJ, Delft, The Netherlands

A nanometre scale pore in a solid state membrane provides a new way to electronically probe the structure of single linear polymers, including those of biological interest in their native environments. Previous work with biological protein pores wide enough to pass and sense single stranded DNA molecules demonstrates the power of the nanopore approach, but many future tasks and applications call for a robust solid-state pore whose nanometre scale dimensions and properties may be selected, as one selects the lenses of a microscope. Here we demonstrate a solid-state nanopore microscope capable of observing individual molecules of double stranded DNA and their folding behaviour. We discuss extensions of the nanopore microscope concept to alternative probing mechanisms and applications including the study of molecular structure and sequencing.

Probing, characterizing and manipulating single biopolymers like DNA is often accomplished with the aid of optical methods, e.g. observing evanescent field fluorescence of dye molecules ${ }^{1}$, deflecting light beams in atomic force microscopes ${ }^{2,3}$ or trapping attached dielectric objects with optical tweezers ${ }^{4,5}$. There has also been remarkable progress at the molecular level in the study of the electrical ionic 
conduction signals from voltage biased nanoscale biopores ${ }^{6,7}$. More recently, a voltage bias on an alpha hemolysin biopore has been shown to induce charged single-stranded DNA and RNA molecules to translocate through the pore ${ }^{8-10}$. Each translocating molecule blocks the open pore ionic current providing an electrical signal that depends on several characteristics of the molecule. This system has limits for studies of biological molecules: the pore is of a fixed size, and its stability and noise characteristics are restricted by chemical, mechanical, electrical, and thermal constraints. These difficulties may be overcome with the use of a suitable solid-state nanopore $^{11}$. Here we report on a solid-state nanopore "microscope" capable of electronically characterizing single long chain polymers like DNA molecules. We also show the first observation of molecule induced quantized current blockades that reveal the folding configuration of single molecules as they pass through the nanopore.

At its heart the microscope consists of a voltage biased nanopore, fabricated in a silicon nitride membrane. The membrane separates two chambers of conducting electrolyte solution. The only electrical conduction path from one chamber to the other passes through the nanopore. To resolve interesting molecular structure the nanopore dimensions must be small enough to avoid averaging over continuous single molecule configurations induced by thermal fluctuations and large enough to pass the smallest dimensions of the molecule to be probed. For double stranded DNA (ds DNA) this means a pore diameter and membrane thickness smaller than the molecule persistence length, $50 \mathrm{~nm}$ for ds DNA, and a pore diameter larger than the $\sim 2 \mathrm{~nm}$ cross sectional size of the molecule. The recent discovery of ion beam sculpting ${ }^{11}$ allows structures that meet these criteria to be fabricated with desired nanometer scale dimensions from solid state materials like silicon nitride. A transmission electron micrograph of an ion sculpted $3 \mathrm{~nm}$ nanopore in a 5-10 $\mathrm{nm}$ thick membrane is shown in Figure 1a, and a schematic of the experimental setup is shown in Figure 1b. 
Open pore ionic conduction was first established with $120 \mathrm{mV}$ bias across the nanopore. Then DNA was added to the negative cis chamber and current blockades appeared in the form of isolated transient reductions in current flow through the pore. Figure 1c shows part of a current trace recorded for $3 \mathrm{~kb}$ DNA $(\sim 1 \mu \mathrm{m}$ long) and a $3 \mathrm{~nm}$ pore. Each event is the result of a single molecular interaction with the nanopore and is characterized by its time duration $\mathrm{t}_{\mathrm{d}}$ and its current blockage, $\Delta \mathrm{I}_{\mathrm{b}}, \sim 120 \mathrm{pA}$. The expected current blockage from a single molecule blocking the pore is linearly dependent on the cross-sectional area of the molecule and independent of the area of the pore, although because the blockage current varies inversely with the thickness of the pore, different pores may produce different blockage currents for the same molecule. Occasionally, the baseline level shifted for very long periods of time by a magnitude similar to that belonging to the discrete transient molecular event. This was likely due to a single molecule that became "stuck" in the nanopore. We do not present any molecular data here for times intervals where the open pore current had been reduced in such a way from its initial value.

Figure 2 shows two plots of the density distribution from many transient molecular events over the parameters $\left\langle\Delta \mathrm{I}_{\mathrm{b}}\right\rangle$ and $\mathrm{t}_{\mathrm{d}} . \quad\left\langle\Delta \mathrm{I}_{\mathrm{b}}\right\rangle$ is defined as the average value of a current blockade over $t_{d}$ (regardless of the signal's shape). Figure $2 \mathrm{a}$ is obtained from experiments using $10 \mathrm{kbp}$ ds DNA with a $3 \mathrm{~nm}$ pore and Figure $2 \mathrm{~b}$ from experiments with $10 \mathrm{kbp}$ ds DNA and a $10 \mathrm{~nm}$ pore. The voltage bias across the pore in both cases was $120 \mathrm{mV}$. The color coding is keyed to the local density of events normalized by the total number of events for each case. Although both distributions peak at $t_{d} \sim 300-400 \mu \mathrm{sec}$ the distribution in $t_{d}$ is quite broad for the $3 \mathrm{~nm}$ pore experiment and much sharper for the $10 \mathrm{~nm}$ pore experiment. On the other hand the distribution of events in $\left\langle\Delta \mathrm{I}_{\mathrm{b}}\right\rangle$ for the $10 \mathrm{~nm}$ pore is much broader than for the $3 \mathrm{~nm}$ pore, with larger $\left\langle\Delta \mathrm{I}_{\mathrm{b}}\right\rangle$ events showing a definite trend towards having smaller values of $t_{d}$. 
A visual study of individual events for the $3 \mathrm{~nm}$ pore plotted in Figure 2a shows them all to be simple single level current blockades of the type at the bottom of Figure 1c (see also inset Figure 4a). Approximately $60 \%$ of the events in $10 \mathrm{~nm}$ pore experiments are of this type but the remainder are more complex (see inset Figure $4 b$ ). Selecting simple single level events from the $10 \mathrm{~nm}$ pore data significantly sharpens the distribution in both $\left\langle\Delta \mathrm{I}_{\mathrm{b}}>\right.$ and $\mathrm{t}_{\mathrm{d}}$. In Figure 3 we present a histogram of $\mathrm{t}_{\mathrm{d}}$ values for simple events in three experiments using $10 \mathrm{~nm}$ pores: $3 \mathrm{kbp}$ ds DNA with $120 \mathrm{mV}$ bias, $10 \mathrm{kbp}$ ds DNA with $120 \mathrm{mV}$ bias, and $10 \mathrm{kbp}$ ds DNA with $60 \mathrm{mV}$ bias. The 10 kbp DNA is seen to take slightly more than 3 times longer to negotiate the pore than the $3 \mathrm{kbp}$ DNA at the same bias. Reducing the bias by a factor of two approximately doubles the translocation time. These observations provide strong evidence that each simple single level event corresponds to a DNA molecule translocating in single file order through the nanopore under the influence of electrophoretic forces. We shall see that the structure of the more complex signals confirms this interpretation.

Figure $4 \mathrm{a}$ shows the density plot of the simple translocation events for $10 \mathrm{kbp}$ DNA passing through the $10 \mathrm{~nm}$ pore. The main cluster of events is narrowly distributed in both $\left\langle\Delta \mathrm{I}_{\mathrm{b}}\right\rangle$ and $\mathrm{t}_{\mathrm{d}}$. We will discuss the second cluster later, but note its mean $<\Delta \mathrm{I}_{\mathrm{b}}>$ is twice that of the main cluster while its mean $\mathrm{t}_{\mathrm{d}}$ is half. Characteristic time recordings of events from these two regions of the density plot are shown in the inset.

Figure $4 \mathrm{~b}$ shows a density plot for more complex "multi-level" events that remain after the simple ones are subtracted. Examples of event time recordings in this group are shown in the inset. They look like simple events on which additional blockade structure has been superimposed. For $\sim 85 \%$ of the complex events the additional structure appears at the front of the event, $\sim 5 \%$ at the rear, $1-2 \%$ at both the front and rear, and $5 \%$ in the middle. Half of the events with structure in the middle have 
$t_{d}>400 \mu \mathrm{sec}$. (More complex structures are also observed in longer $t_{d}$ events.) We attribute these remarkable additional features to DNA molecules that are folded on themselves as they pass through the pore. As overlapping folded parts of a molecule pass through the pore they enhance the current blockade during that part of the event. If the instantaneous current blockade is proportional to the number of strands of the same molecule in the pore (i.e. one or two), one calculates that the average current blockade for the event will be inversely proportional to the translocation time $t_{d}$,

$$
<\Delta \mathrm{I}_{\mathrm{b}}>=<\Delta \mathrm{I}_{0}>\mathrm{t}_{0} / \mathrm{t}_{\mathrm{d}}
$$

where $\left\langle\Delta \mathrm{I}_{0}\right\rangle$ and $\mathrm{t}_{0}$ are the mean current blockage and translocation time of a simple event. This simple model, plotted as the dotted line in Figure 4b, shows excellent agreement with the data. The smaller cluster in Figure $4 \mathrm{a}$ is thus interpreted as due to molecules that are folded nearly in the middle of the strand. Residual closed circle plasmid DNA in the sample preparation could presumably contribute to this peak.

More confirmation that complex nanopore signals correspond to events where folded DNA molecules translocate through the pore is provided by a study of the distribution of instantaneous blockade current magnitudes over all events. Assuming the instantaneous magnitude of the blocked current is in proportion to the instantaneous number of strands of ds DNA in the nanopore, we expect the distribution of blocked currents taken over many events, in time samples much smaller than an event duration, to show a quantization of local instantaneous $\Delta \mathrm{I}_{\mathrm{b}}$ values corresponding to $0,1,2, \ldots$ strands of the folded molecule in the pore at any particular time. A histogram of these sampled values of $\Delta \mathrm{I}_{\mathrm{b}}$ for $10 \mu \mathrm{sec}$ samples over $\sim 9500$ events (including $200 \mu \mathrm{sec}$ before and after each event) is shown in Figure $5 \mathrm{a}$ for the $10 \mathrm{~kb}, 120 \mathrm{mV}$ data and in Figure $5 \mathrm{~b}$ for the $10 \mathrm{~kb}, 60 \mathrm{mV}$ data. The expected quantization of sampled $\Delta \mathrm{I}_{\mathrm{b}}$ values is clearly seen corresponding to zero, one and two molecule strands occupying the 
nanopore (note the log scale). Experiments with $50 \mathrm{kbp}$ DNA and a 15 - $20 \mathrm{~nm}$ pore (data not shown) also show three level blockades.

A molecule microscope based on solid-state nanopore provides distinct differences and/or advantages over extant biopore detectors. All results presented here were for ds DNA molecules whose transverse size is $\sim 2 \mathrm{~nm}$. The hemolysin biopore used in previous studies cannot translocate such a large diameter molecule ${ }^{8,12}$. We emphasize that because solid-state nanopores can now be fabricated with arbitrary size apertures the nanopore microscope enables the study of single molecules in a vast category of unlabelled polymers in solution, including RNA, hybridized DNA, and proteins. Furthermore, because of their physical robustness, solid-state nanopores may well be used to study molecules at extremes of temperature, voltage, and $\mathrm{pH}$ conditions that would destroy biopore-membrane systems.

The average speed of DNA molecules translocating through $120 \mathrm{mV}$ biased 10 $\mathrm{nm}$ pores is $\sim 1 \mathrm{~cm} / \mathrm{sec}$. A quantitative understanding of this result can involve many complex issues like hydrodynamic interactions and screenings, electro-osmotic flow in and near the pore, and non-equilibrium statistical considerations ${ }^{14-17}$. Here we note that a simple combination of likely relevant parameters, derived by equating the electric force on the charged polymer in the pore to a viscous drag on an effective sphere of radius $a$ on either side of the pore, gives an average translocation speed of

$$
v=C \frac{\sigma V_{\text {bias }}}{(2 a)(6 \pi \eta)}
$$

where $\sigma$ is the linear charge density on the molecule, $\eta$ the viscosity of the solution, $\mathrm{V}_{\text {bias }}$ the pore voltage bias and $\mathrm{C}$ is a factor of order unity accounting for the complex 
issues mentioned above. Setting $a$ to the persistence length of $\mathrm{DNA}^{18}$ (which implies statistical loss of effective drag force beyond that distance) and assuming a charge of e/3 per phosphate, we find that $\mathrm{C} \sim 1 / 2$ brings equation (2) in line with the experimental observations. This crude result suggests that understanding translocation speed may well require only a mesoscopic fluid dynamical description without the need for invoking strong chemically specific complex interactions between molecule and pore. The agreement also strengthens the notion that the observed signals correspond to molecular translocations.

The observed structured events provide additional compelling evidence in favor of translocation. The quantization of current blockage levels shows that molecules must be completely threading the pore (if molecules were partially blocking the pore, one would expect a continuous range of blockade currents corresponding to different degrees of pore occlusion). The large electric force ( $8 \mathrm{kT} / \mathrm{nm}$ for $120 \mathrm{mV}$ bias) on a ds DNA molecule in the pore ensures that a molecule that fully enters the pore will translocate. Finally, the fact that deeper blockages correspond to shorter events and the good agreement of the data with equation (1) are consistent only with folded molecules translocating the pore. Although we have previously shown that a solid state nanopore can be used to detect the presence of DNA molecules ${ }^{11}$, these new observations, coupled with the aforementioned length and bias dependent measurements, represent the first time individual molecules have been shown to translocate the kind of solid state pore needed for a nanopore microscope.

A new feature of the solid-state nanopore microscope is its ability to detect molecular folding. The majority of leading edge folds might be expected to result from a molecule initially encountering the pore at some distance from its end with the 
electrophoretic force from the pore forcing a fold as the translocation starts. Simple energetic considerations using the charge on the molecule and its elastic constant show that this is quite possible for the electric fields and pore sizes in our experiments. This does not explain the trailing edge folds which we ascribe to the pre-existing state of the molecule (before translocation). The intermediate size $10 \mathrm{~nm}$ pore presented here offers the new ability to observe this folding. Smaller pores (e.g. our $3 \mathrm{~nm}$ pore and hemolysin pores) do not allow the passage of a folded molecule, while other constrictions recently demonstrated $^{19,20}$ are much larger than the persistence length of DNA and so do not restrict the passage of DNA to a discrete set of measurable conformations. Figure $2 b$ shows evidence for attractive interactions between different molecules that very occasionally pair up to provide single translocation events for two connected molecules. These appear as a "ghost" structure at twice the expected translocation times. Although very few, these events form a separate cluster clearly seen in the figure. There are too many of these events to be explained as a simple consequence of the Poisson distribution of event arrivals.

It's important to note that much of our discussion of the solid state nanopore microscope results has focused on understanding the properties of distributions of molecular events. But that understanding has ultimately enabled us to interpret time histories of individual molecules -- and their structures -- as they pass through the nanopore in terms of the corresponding highly modulated individual events in the inset of Figure 4b. We have thus demonstrated the ability of the solid state nanopore microscope to resolve multiple features on a single molecule without a priori knowledge of the feature's characteristics and to observe new phenomena, like molecular folding and pairing by means that have not been available in narrower existing biopores. This significantly augments previous work showing that biological 
pores can distinguish between molecules whose chemical structures ${ }^{9,21}$ provide selective binding to a biopore pore, and work that shows biological pores can trap and sense the spontaneous disassociation of carefully prepared DNA hairpins ${ }^{22}$ that are frustrated from translocating the biopore in their associated form. The solid state pores provide a new way to study the folding and pairing configurations of single long chain molecules, the differences between chemically identical molecules in a statistical ensemble and induced changes in molecular structure that, because of energy restrictions, do not occur naturally in solution.

Finally we mention that a major advantage of a microscope based on solid-state pores lies in the possibility of articulating the nanopores with electrically conducting electrodes. Such electrodes can allow electronic tunneling and near field optical studies of translocating molecules that are linearized and confined in a nanopore of the microscope. Applying these new physical local interactions to molecules translocating through nanopores can provide local single molecule spectroscopies not afforded by measurement of ionic current alone and offer a means of increasing longitudinal resolution, possibly to the single-base level for DNA, allowing for extremely rapid sequencing of long molecules. It does not seem far fetched to believe that a mastery of the materials science, surface physics, fluid mechanics, and electrical and optical properties of solid-state nanopores will lead to dramatic improvements in nanopore microscopes' ability to probe important biological molecules.

\section{Methods}

Nanopores used in our "microscope" were fabricated in $25 \mu \mathrm{m} \times 25 \mu \mathrm{m}$ free standing silicon nitride membranes supported by $3 \mathrm{~mm}$ x $3 \mathrm{~mm}$ x $0.3 \mathrm{~mm}$ silicon substrate (100) frames. The $500 \mathrm{~nm}$ thick, low stress ( $200 \mathrm{MPa}$ tensile) silicon nitride membrane was deposited by low pressure chemical vapor deposition. Photolithography and anisotropic 
wet chemical etching of silicon were used to create the free standing $\mathrm{SiN}$ membrane. An initial $0.1 \mu \mathrm{m}$ diameter pore was created at the membrane's center using a focused ion beam (FIB, Micrion 9500) machine. The diameter of this large pore was then decreased to molecular size near one surface of the membrane using feedback controlled ion beam sculpting ${ }^{11}$. The final nanopore thus resides in a thin 5-10 $\mathrm{nm}$ thick membrane that covers an approximately $0.1 \mu \mathrm{m}$ diameter cylindrical aperture that extends through the thick silicon nitride membrane below. Nanopore diameters were determined by transmission electron microscopy. We note that because the TEM projects a three dimensional structure on to a two dimensional plane, the image of the inner edge of the pore actually represents the minimum projected diameter of the pore wall at any height and may not correspond to the narrowest physical constriction. Also, because of the inherent inaccuracies of TEM for determining absolute size and the fact that our pores are not perfectly round, all sizes should be taken as estimates to within a nanometre of the actual pore size.

Figure $1 \mathrm{~b}$ shows a diagram of the "nanpore microscope apparatus." The nanopore on the silicon chip separates two chambers filled with buffered salt solution (1M KCl, 10mM TRIS-HCl, $\mathrm{pH} 8.0$ ). Pre-soaking the chip in isopropanol was found to aid wetting the pore. The cis chamber, to which DNA molecules are added, is at the top of the figure and the trans chamber at the bottom. Both chambers are made of PDMS (polydimethylsiloxane) and are equipped with $\mathrm{AgCl}$ electrodes across which a voltage bias is applied during experiments. The electrode in the trans chamber is positively biased and connected to the current sensing electronics, while the other electrode is connected to signal ground.

Double-stranded (ds) DNA with $\sim 3$ kilobase-pairs (kbp) and $10 \mathrm{kbp}$ were used in this work. The 3kbp DNA was prepared from pUC19 plasmid (New England 
Biolabs). The plasmid was cleaved at a single site with SmaI restriction enzyme to produce blunt-ended linear double-stranded DNA. The purity and quantity of the recovered DNA following phenol extraction were assessed by agarose gel electrophoresis and UV absorbance. The $10 \mathrm{kbp} \mathrm{KBA}$ plasmid ${ }^{23}$ was linearized by digestion with the SmaI and purified following agarose gel electrophoresis using the QIAquick gel extraction kit (QIAGEN Inc., Valencia, CA). The DNA was concentrated by ethanol precipitation as described by Sambrook, et. al. (Molecular Cloning; a laboratory manual, Cold Spring Harbor) and stored dry at 4 C. Dried DNA was suspended in $10 \mathrm{mM}$ Tris, $1 \mathrm{mM}$ EDTA $\mathrm{pH} 7.6$ (RT) prior to use. Typical concentrations of DNA in the cis chamber were $\sim 10 \mathrm{nM}$.

Ionic current through the solid-state nanopore was measured and recorded using an Axopatch 200B integrating patch clamp amplifier system (Axon Instrument) in resistive feedback mode. Signals were preprocessed by a $10 \mathrm{kHz}$ low pass filter. Except for the data displayed in figure 1c, which is a live recording, all data was acquired in event driven acquisition mode, meaning analogue start and stop triggers were used to determine when data was to be recorded.

1. Levene, M.J., Korlach, J., Turner, S.W., Foquet, M., Craighead, H.G., \& Webb, W.W. Zero-Mode Waveguides for Single-Molecule Analysis at High Concentrations. Science, 299, 682-686 (2003).

2. Rees, W.A., Keller, R.W., Vesenka, J.P., Yang, G. \& Bustamante, C. Evidence of DNA Bending in Transcription Complexes Imaged by Scanning Force Microscopy. Science, 260, 1646-1649 (1993).

3. Hansma, H.G., Kim, K.J., Laney, D.E., Garcia, R.A., Argaman, M., Allen, M.J., \& Parsons, S.M. Properties of Biomolecules Measured from Atomic Force Microscope Images: A Review. J Struct Biol. 119, 99-108 (1997). 
4. Quake, S.R., Babcock, H. \& Chu, S. The Dynamics of Partially Extended Single Molecules of DNA. Nature, 388, 151-154 (1997).

5. Smith, S. B., Cui, Y. \& Bustamante, C. Overstretching B-DNA: The Elastic Response of Individual Double-Stranded and Single-Stranded DNA Molecules. Science, 271, 795-799 (1996).

6. Hille, B. Ionic Channels and Excitable Membranes. (Sinauer, Sunderland, Massachusetts, 1992).

7. Bezrukov, S.M., Vodyanoy, I., \& Parasegian, V.A. Counting polymers moving through a single ion channel. Nature, 370, 279-281 (1994).

8. Kasianowicz, J. J., Brandin, E., Branton, D. \& Deamer, D.W. Characterization of individual polynucleotide molecules using a membrane channel. Proc. Natl. Acad. Sci. U.S.A., 93, 13770-13773 (1996).

9. Akeson, M., Branton, D., Kasianowicz, J.J., Brandin, E. \& Deamer, D.W. Microsecond time-scale discrimination among polycytidylic acid, polyadenylic acid, and polyuridylic acid as homopolymers or as segments within single RNA molecules. Biophys. J., 77, 3227-3233 (1999).

10. Meller, A., Nivon, L., Brandin, E., Golovchenko, J., \& Branton, D., Rapid Nanopore Discrimination Between Single Polynucleotide Molecules, Proc. Natl. Acad. Sci. 97,1079-84 (2000).

11. Li, J., Stein, D., McMullan, C., Branton, D., Axix, M.J., \& Golovchenko, J.A. Ion-beam sculpting at nanometre length scales. Nature, 412, 166-169 (2001).

12. Sauer-Budge, A.F. Unzipping double-stranded DNA molecule by molecule in a nanopore. (PhD. Thesis, Harvard University, Cambridge, Massachusetts, 2002).

13. Lubensky, D.K., \& Nelson, D.R. Driven Polymer Translocation Through a Narrow Pore. Biophys. J., 77, 1824-1838 (1999). 
14. Muthukumar, M. Dynamics of Polyelctrolyte Solutions, J. Chem Phys. 107, 2619-2635 (1997).

15. Muthukumar, M. Polymer Translocation Through a Hole, J. Chem. Phys. 111, 10371-10374 (1999).

16. Muthukumar, M. Polymer escape through a Nanopore, J. Chem. Phys, 118, 5174-5184 (2003).

17. Lee, N., \& Obukhov, S. Diffusion of a Polymer Chain Through a Thin Membrane, Journal de Physique II, 6, 195-204 (1996).

18. Evans, D.F. \& Wennerstrom, H. The Colloidal Domain. (Wilry-VCH, New York, 1999) p. 361.

19. Han, J. \& Craighead, H. G. Separation of Long DNA Molecules in a Microfabricated Trap Array. Science, 288, 1026-1029 (2000).

20. Saleh, O.A. \& Sohn, L.L. An Artificial Nanopore for Molecular Sensing. Nano Letters, 3, 37-38 (2003).

21. Kasianowicz, J.J., Henrickson, S.E., Weetall H.H., and Robertson, B. Simultaneous Multianalyte Detection With a Nanopore. Analytical Chemistry, 73, 2268-2272 (2001).

22. Vercoutere, W., et al. Rapid Discrimination Among Individual DNA Hairpin Molecules at Single-Nucleotide Resolution Using an Ion Channel. Nature Biotechnology, 19, 248-252 (2001).

23. Byers, T.J., Husain-Chishti, A., Dubreuil, R.R., Branton, D., \& Goldstein, L.S. Sequence Similarity of the Amino-Terminal Domain of Drosophila Beta Spectrin to Alpha Actinin and Dystrophin, J Cell Biol, 109, 1633-1641 (1989).

We wish to acknowledge valuable discussion and criticism of this work by Professors Daniel Branton, Murugappan Muthukumar, and Michael Aziz. Dr. Hui Wang prepared the $3 \mathrm{kbp}$ DNA used in the work. 
Professor Alec Kavcic, Dr Michael Burns, Albert Huang and Jiajun Gu assisted with software analysis. Professor Qun Cai assisted with nanopore preparation and Christopher Russo provided assistance during preparation of this manuscript. Support for this research has been provided by DARPA, NSF, DOE, AFOSR and Agilent Technologies

Correspondence and requests for materials should be addressed to J. A. G. (e-mail: golovchenko@physics.harvard.edu.).

Figure 1. Details of the experimental setup. a) TEM image of a $\sim 3 \mathrm{~nm}$ silicon nitride nanopore. b) Schematic of solid-state nanopore microscope used to obtain electrical signals from single DNA molecules. c) Characteristic signals showing transient molecular current blockades and a baseline current shift. Parameters $t_{d}$ and $\left\langle\Delta l_{b}\right\rangle$ are shown for a selected simple molecular event.

Figure 2. Distribution of events as a function of $t_{d}$ and $\left\langle\Delta \mathrm{l}_{b}>\right.$ for $10 \mathrm{kbp}$ DNA with a) a $3 \mathrm{~nm}$ pore, 2674 events, and b) with a $10 \mathrm{~nm}$ pore, 9477 events. The bias voltage was $120 \mathrm{mV}$. The color scale represents event fraction density normalized as a probability distribution so that the integral of the density over all $t_{d}$ and $\left\langle\Delta I_{b}>\right.$ is equal to 1 .

Figure 3. Plot of the translocation time distribution function for $3 \mathrm{kbp}$ and $10 \mathrm{kbp}$ DNA molecules in a $10 \mathrm{~nm}$ nanopore at $120 \mathrm{mV}$ bias, and for $10 \mathrm{kbp}$ DNA at 60 $\mathrm{mV}$ bias.

Figure 4. Density of events over $t_{d}$ and $\left\langle\Delta l_{b}>\right.$ where plot a) contains simple events characterized by a single blockade level, and plot b) contains the remaining complex events. The inserts show examples of blockade current time traces of events that contribute to the density plots. Isolated regions with 
only one event in a $20 \mu \mathrm{sec}$ by $2 \mathrm{pA}$ bin are not displayed in this plot. The color scale is the same as in Figure 2. The dotted line in b) represents the prediction of equation (1) in the text with $\mathrm{t}_{0}=400 \mu \mathrm{sec}$ and $\left\langle\Delta \mathrm{I}_{0}\right\rangle=100 \mathrm{pA}$.

Figure 5. Plot of the instantaneous time distribution of blockade current $\Delta \mathrm{l}_{\mathrm{b}}$ over all events. Current is sampled in a 10 microsecond time window. The quantized blockade currents corresponding to 0,1 and 2 strands in the pore are clearly seen for $10 \mathrm{kbp}$ DNA data from a $10 \mathrm{~nm}$ pore for both $120 \mathrm{mV}$ bias and $60 \mathrm{mV}$ bias. 


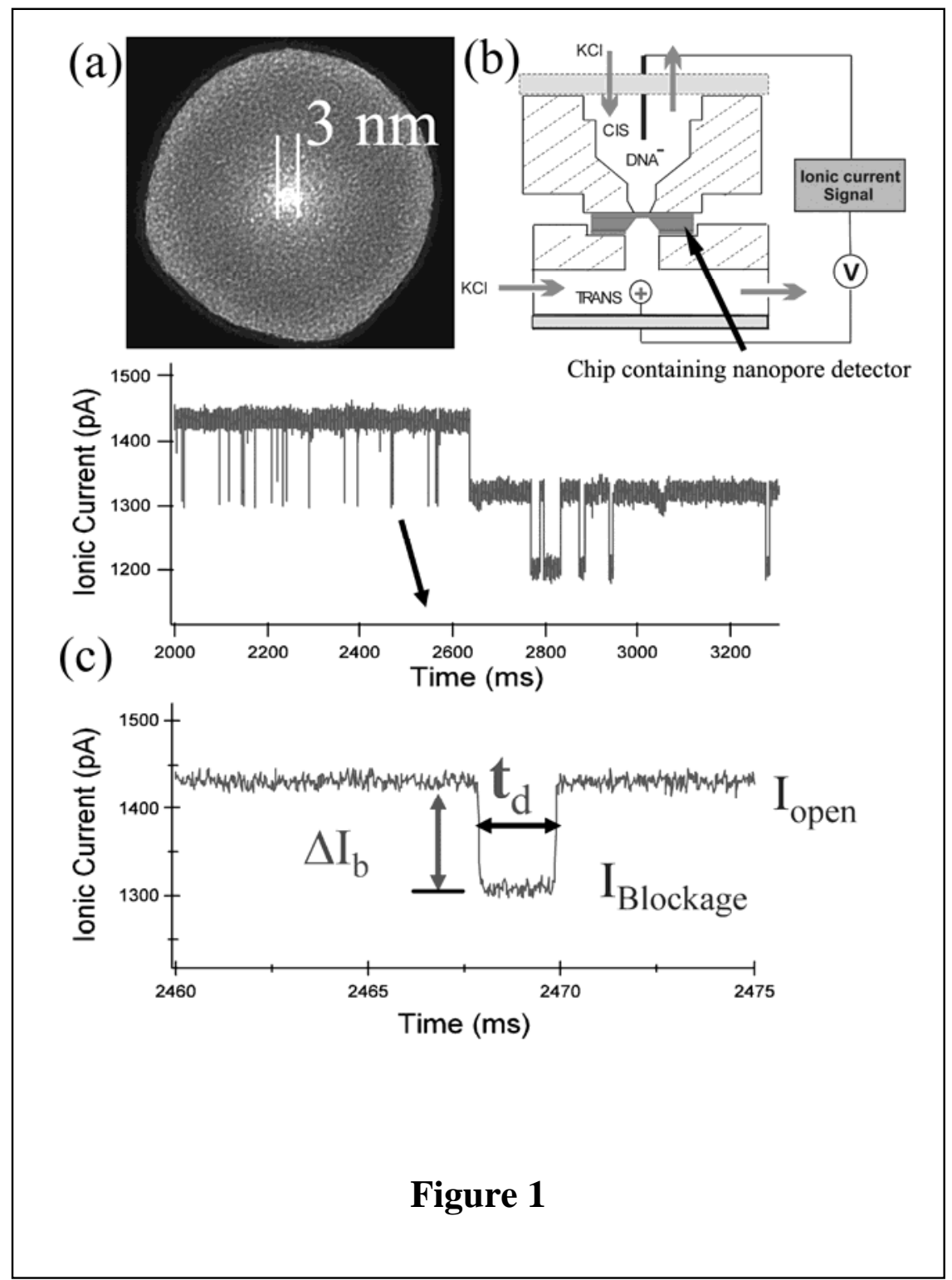




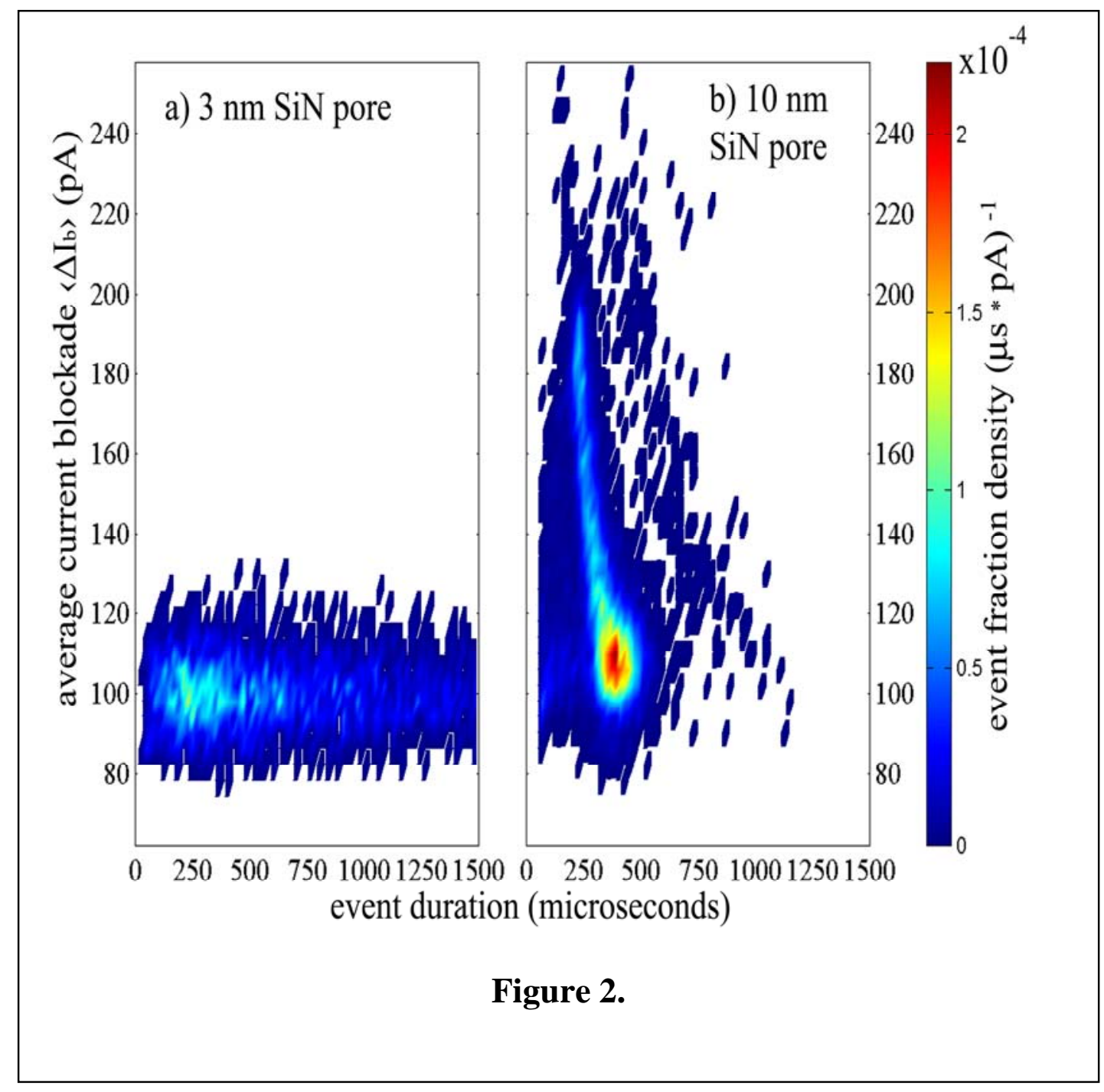




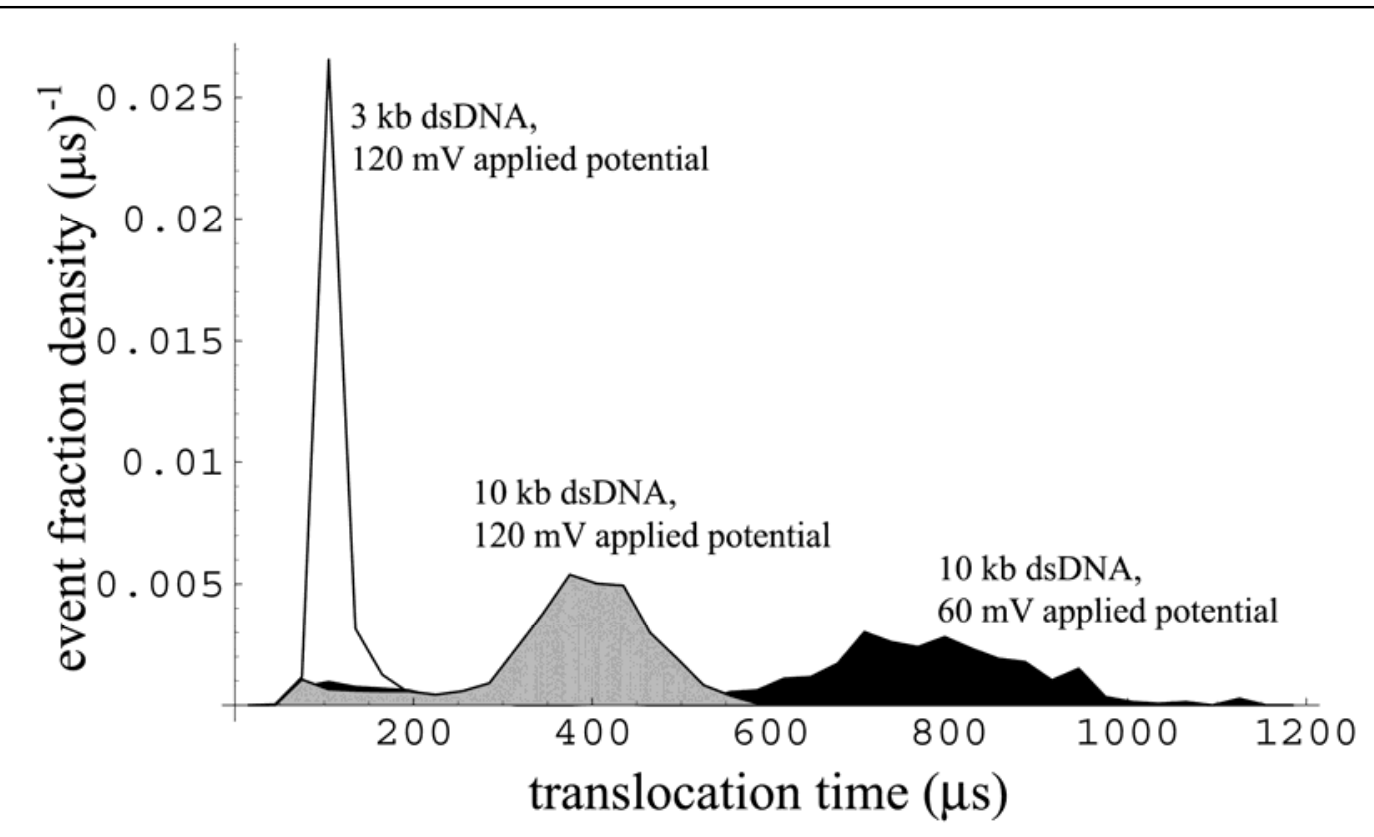

Figure 3. 


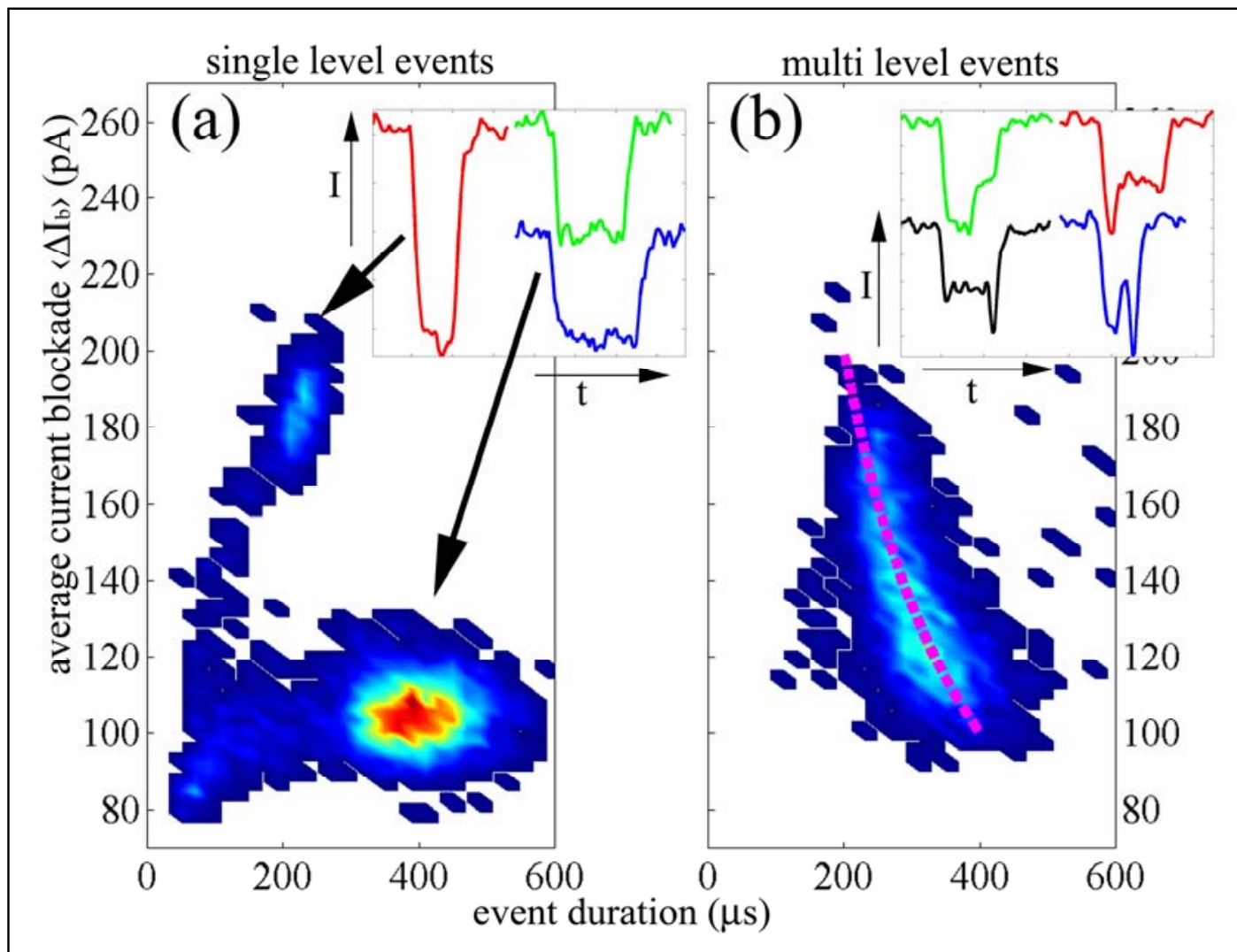

Figure 4. 


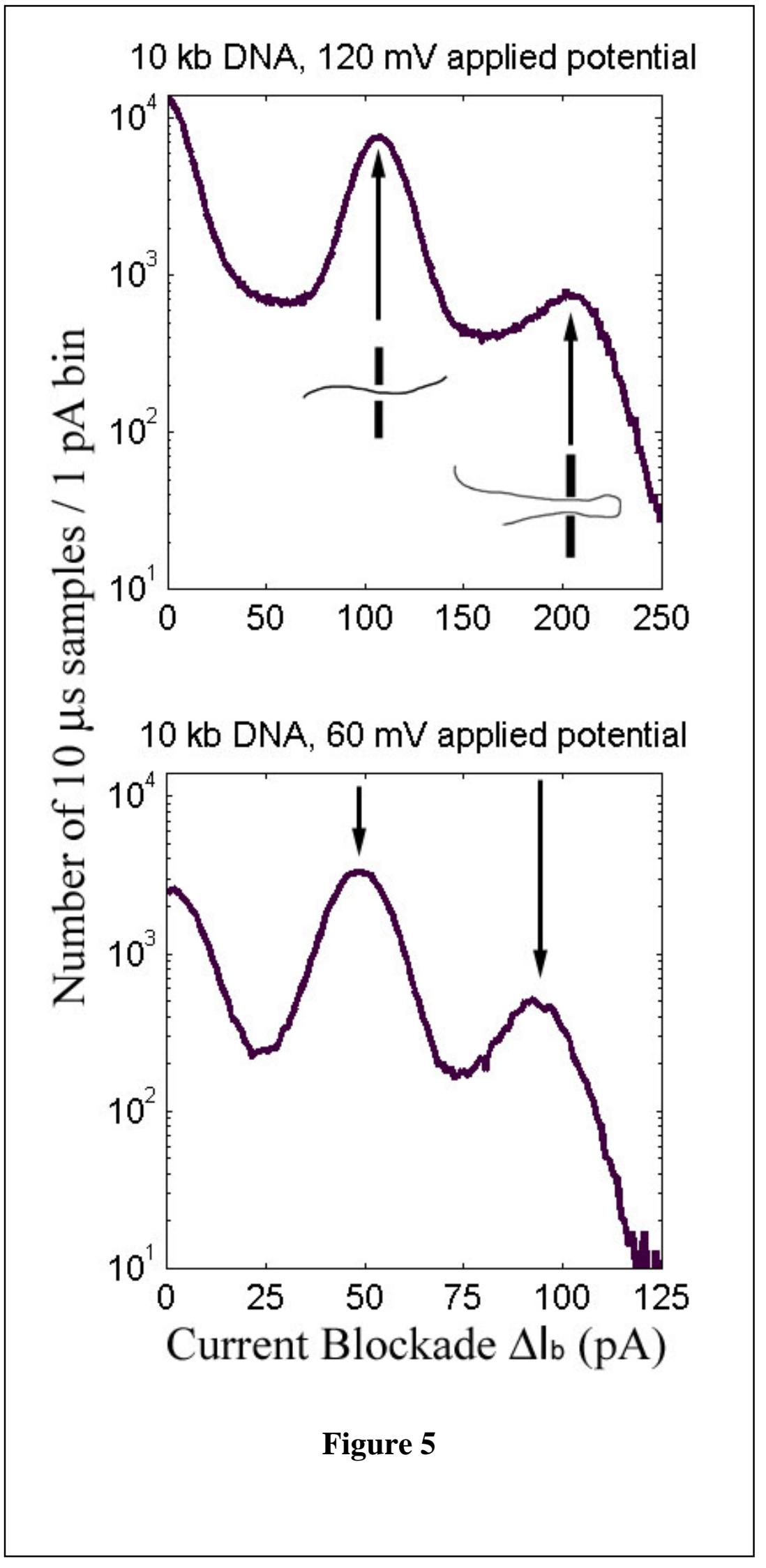

